\title{
Granuloma inguinale of the cervix: a carcinoma look-alike
}

\author{
A A Hoosen, G Draper, J Moodley, K Cooper
}

\begin{abstract}
Granuloma inguinale of the cervix presents as a proliferative growth and may mimic carcinoma. Over a 3 year period 18 patients with cervical granuloma inguinale were detected. Nine $(50 \%)$ had a clinical diagnosis of carcinoma of the cervix on admission, five of whom had extensive investigations to exclude a malignancy. In communities where these two conditions are commonly seen it is recommended that granuloma inguinale be considered in young women with granulomatous lesions of the cervix, so as to avoid extensive and financially costly investigations, and unnecessary psychological stress for patients.
\end{abstract}

Carcinoma of the cervix is a common condition in South Africa. Recently this malignancy has been reported to occur more frequently in younger women. ${ }^{1}$ In tropical and sub-tropical regions, young sexually active women are also prone to develop granulomatous genital conditions such as tuberculosis, amoebiasis, schistosomiasis and granuloma inguinale. Granuloma inguinale, in particular, may present as a proliferative growth on the cervix and may easily be mistaken for carcinoma. It is therefore, important to exclude this benign and easily treatable condition in any young women thought to have cervical carcinoma.

We present a series of 18 women with cervical granuloma inguinale. In nine a clinical diagnosis of carcinoma of the cervix was made at initial presentation.

\section{Patients and methods}

Hospital and laboratory records covering a 3 year period were reviewed for patients with cervical granuloma inguinale. These revealed a total of 18

Department of Medical Microbiology, Faculty of Medicine, University of Natal, Durban, South Africa A A Hoosen

Department of Obstetrics and Gynaecology, Anatomical Pathology, Faculty of Medicine, University of Natal, Durban, South Africa

G Draper, J Moodley, K Cooper patients with histologically confirmed disease. All biopsied specimens were fixed in formalin and sections were routinely stained with haematoxylin and eosin stain. Warthin-Starry silver and Giemsa stains were performed when necessary.

\section{Results}

For the purpose of analysis, these patients were divided into two groups namely Group A, consisting of nine patients $(50 \%)$ in whom a clinical diagnosis of carcinoma of the cervix was made at the initial presentation and Group B consisting of the remaining nine patients in whom other diagnoses were made initially.

The patient characteristics and clinical features are summarised in the table. In three patients, smears from cervical lesions, stained with Giemsa stain, showed typical Donovan bodies in large monocytes. Biopsy specimens were taken from all patients for histological examination. These specimens when stained with haematoxylin and eosin typically showed a mixed infiltrate of polymorphonuclear neutrophils, histiocytes and plasma cells. Some histiocytes were vacuolated and contained characteristic Donovan bodies. The bipolar staining and safety pin appearance of Calymmatobacterium granulomatis was better appreciated with the Warthin-Starry silver and Giemsa stains.

In seven patients, the lesion was seen on the cervix alone, whilst in five, lesions were also present on the vulva. The majority of patients were treated with tetracycline; three pregnant patients received erythromycin and the treatment records for the remaining patients were not available. Most patients showed good initial responses to therapy, but were discharged or referred to peripheral hospitals before lesions were completely healed. Results of serological tests for syphilis were available in nine patients and the test was positive in only one.

Of the nine patients in group $\mathrm{A}$, the initial clinical diagnosis of carcinoma of the cervix was revised in four whilst the remaining five patients were investigated as for carcinoma of the cervix. This included examination under general anaesthesia and cystoscopy for staging of the disease. Three patients were classified as stage II and another 2 as stage III. Intravenous pyelograms were performed on six patients including all five patients in the group 
Table Clinical data on 18 patients with cervical granuloma inguinale

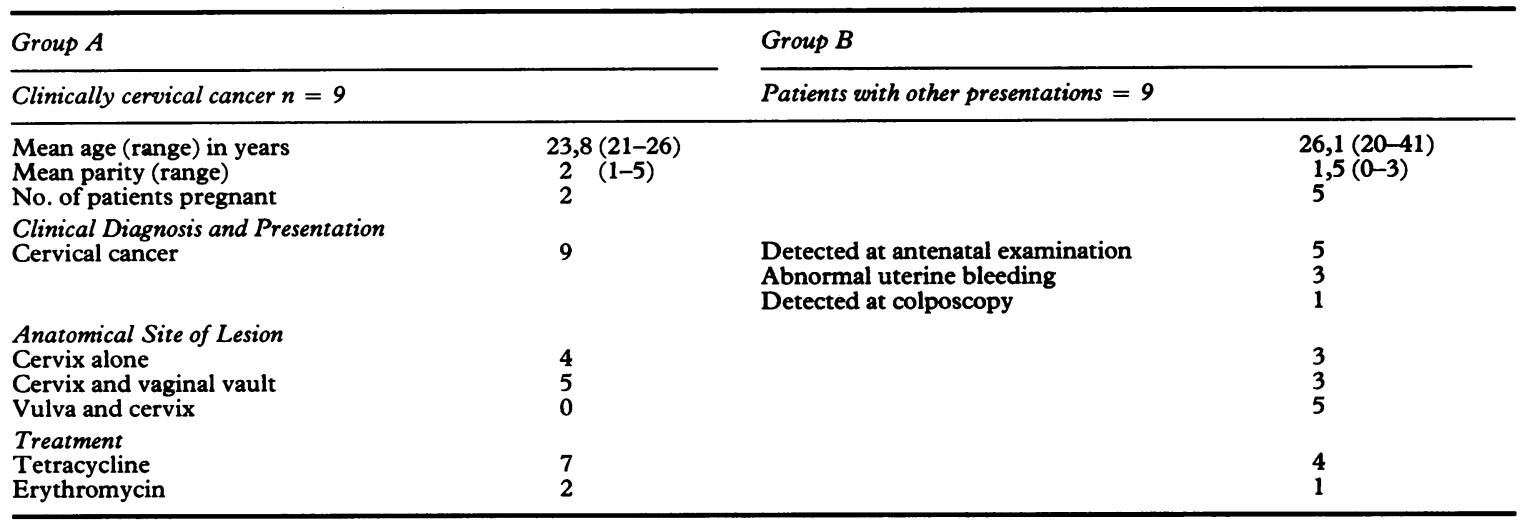

investigated further for carcinoma of the cervix. Obstructive uropathy was noted in four. Hydronephrosis was detected in three resulting in two of the patients originally regarded as having stage II disease being restaged as having stage III carcinoma of the cervix.

\section{Case report}

The following case report illustrates a fairly typical presentation and highlights some of the problems related to diagnosis and management. Ms T G, aged 21 years presented with a one month history of a watery offensive vaginal discharge, intermenstrual bleeding, deep dyspareunia and lower abdominal pain. Clinically she had a large exophytic, friable lesion involving the anterior lip of the cervix. This extended to the upper third of the vagina and involved the parametrium up to the side wall of the pelvis. A clinical diagnosis of carcinoma of the cervix stage IIIA was made.

Relevant investigations included an intravenous pyelogram which showed a nonfunctioning kidney on the right. Examination under anaesthesia confirmed the staging of the disease and cystoscopy revealed schistosomiasis of the bladder which was confirmed histologically. Prior to the histological results of the cervical biopsy being known, the possibility that she may have carcinoma and the reason for her planned visit to the combined gynaecology oncology clinic was discussed with her.

The histological report showed granuloma inguinale and excluded any malignancy of the cervix. The patient was commenced on therapy with oral tetracycline. After a week of treatment the lesion had decreased markedly in size and sonographic examination of the kidneys revealed normal sized kidneys with mild hydronephrosis on the right side. The patient was discharged on tetracycline therapy and asked to return one month later, which she unfortunately failed to do.

\section{Discussion}

Granuloma inguinale is commonly regarded as a disease affecting the vulval and perineal areas. Cervical involvement is well recognised, but is apparently rarely seen in clinical practice. ${ }^{2-4}$ In their study of 351 cases of genital granuloma inguinale, Sengupta and Das ${ }^{4}$ identified 35 cases $(10 \%)$ with cervical involvement. Several reports ${ }^{5-7}$ of granuloma inguinale in South Africa have appeared but only one $^{5}$ mentions three cases in which the cervix was affected. The 18 cases reported in this study were part of a larger group of 39 cases which included all cases of genital granuloma inguinale seen at King Edward VIII Hospital, Durban over the same period of time. The proportion with cervical involvement $(46 \%)$ is much higher than reported elsewhere.

As is the case with carcinoma of the cervix, cervical granuloma inguinale may spread to the parametrial tissue. The fact that obstructive uropathy was detected by IVP in four of six cases of our patients with cervical granuloma inguinale shows the value of this investigation.

Notwithstanding the observed trends in the increasing incidence of cervical carcinoma among younger women, the relative youth of a patient presenting with what looks like carcinoma of the cervix should make one consider other entities such as granuloma inguinale, especially in cases where the lesion is atypical. In the case report presented, the patient was 21 years old and diagnosed as having advanced malignancy (Stage IIIA). This should have alerted one to consider other diagnoses. In such circumstances the use of smears may provide a rapid and useful pointer towards the diagnosis while awaiting histological confirmation. A rapid staining technique $^{8}$ has been demonstrated locally (King Edward VIII Hospital, Durban) and may prove to be of benefit especially in busy gynaecology clinics.

An association between granuloma inguinale and carcinoma of the lower genital tract has been suggested over the years. As yet no confirmatory 
evidence for this association exists. In our series of patients, there were none with co-existing granuloma inguinale and carcinoma of the cervix.

Five of the 18 patients had visible lesions of granuloma inguinale on the external genitalia. Failure to perform a speculum or a digital vaginal examination could result in coexisting cervical, vaginal and pelvic lesions and possible obstructive uropathy being missed. We believe such an examination should be an integral part of the workup of any patient, even if she presents with obvious vulval lesions. This is especially applicable in situations of high patient turnover such as sexually transmitted disease clinics.

Granuloma inguinale has been called a disease "easily forgotten and poorly pursued." "In areas where both carcinoma of the cervix and granuloma inguinale are prevalent there is a need to differentiate rapidly between these diseases to reduce the performance of costly and extensive investigations and avoid unnecessary psychological stress for the patient.
Address for correspondence: A A Hoosen, Department of Medical Microbiology, University of Natal, P.O. Box 17039, Congella 4013 Durban, Republic of South Africa

1 Elliot PM, Tattersall MHN, Coppleson M, et al. Changing character of cervical cancer in young women. $\mathrm{Br} M e d \mathrm{~J}$ 1989;298:288-90.

2 Bhagwandeen SB, Mottiar YA. Granuloma venereum. J Clin Path 1972;25:812-6.

3 Sikazwe NC. Female genital granuloma venereum at University Teaching Hospital, Lusaka. E Afr Med J 1987;64:516-9.

4 Sengupta SK, Das N. Donovanosis affecting cervix, uterus and adnexae. Am J Trop Med Hyg 1984;33:632-6.

5 Freinkel AL, Counihan RJ. Granuloma inguinale (donovanosis) in South Africa. $S$ Afr Med J 1983;63:599-601.

6 Freinkel A. Granuloma inguinale (Donovanosis). $S$ Afr J Sex Transm Dis 1984;4:43-7.

7 Wistrand R, Wegerhoff F. Granuloma inguinale in the Eastern Transvaal. S Afr Med J 1985;67:13-15.

8 O'Farrell N, Hoosen AA, Coetzee KD, van den Ende J. A rapid stain for the diagnosis of granuloma inguinale. Accepted for publication. Genitourin Med 1990;66:200.

9 Growdon WA, Lebherz TB, Moore JG et al. Granuloma inguinale in a white teenager-easily forgotten, poorly pursued. West J Med 1985;143:105-8.

Accepted for publication 5 June 1990 